\title{
Homogenization of the 1D Peri-static/dynamic Bar with Constant Micromodulus
}

\author{
Kjell Eriksson ${ }^{1} \cdot$ Christer Stenström ${ }^{2}$ (D)
}

Received: 6 March 2019 / Accepted: 19 December 2019 / Published online: 10 March 2020

(C) The Author(s) 2020

\begin{abstract}
Because of the nonlocal interparticle forces inherent in peridynamics, surface, boundary, and end effects appear in 3D, 2D and 1D body problems, respectively. In certain situations, the effect is seen as a disturbance, and various efforts, mostly centering on 2D and 1D problems, have been made to reduce it. A simple method has been derived to remove the end effects in a 1D body by homogenizing the body. When a certain body type, common in practice, is homogenized, its linear elastic behavior, independent of the interparticle force range and with a finite number of material points, in the limit infinite, is identical to that of a corresponding classical continuum mechanics body.
\end{abstract}

Keywords Peridynamics $\cdot$ Peristatics $\cdot$ Homogenization $\cdot$ Nonlocal methods

\section{Introduction}

Peridynamic theory, introduced by Silling [1], is a nonlocal extension of solid mechanics, in which each material point is connected to its neighboring material points through pairwise forces acting inside a closed horizon. The formulation allows the handling of long-range forces in general and the unguided modeling of fractures in particular [1, 2]. Peridynamics is based on integral equations that only involve the displacement field, thereby avoiding the spatial derivatives that do not exist across discontinuities in classical continuum mechanics.

The pairwise forces of two material points are related to displacement through the stiffness of the bond between the points. The bond stiffness is computed under the assumption that a material point has a complete neighborhood. However, the neighborhood of a point near a boundary is incomplete, and using the complete neighborhood bond stiffness in a boundary region, results in a softer material and larger strains in such regions.

Kjell Eriksson

kjell.eriksson@1tu.se

Christer Stenström

christer.stenstrom@1tu.se

1 Mechanics of Solid Materials, Luleå University of Technology, Luleå, Sweden

2 Operation and Maintenance Engineering, Luleå University of Technology, Luleå, Sweden 
For a 1D peristatic/dynamic bar, this effect appears near the ends of the bar but affects the entire bar. In general, a very large number of material points, in the order of 100-1000, is required to simulate a homogeneous bar, i.e., to achieve a displacement field that does not deviate notably from the analytical solution of a continuum mechanics bar.

In this paper, by using the stiffness matrix formulation, we derive the parameters necessary to adjust the bond stiffness between material points near the ends of a fully homogenized 1D bar. The method represents a first step in homogenization in peridynamics in general.

The micromodulus $c$, the elastic stiffness of the bond between two material points, was derived by Bobaru et al. [3] for continuous bars, i.e., bars with an infinite number of points inside the horizon. We derive the ensuing conditions for bars with a finite number of points inside the horizon.

The 1D bar has been studied over the years as a natural branch of peridynamic theory. Silling et al. [4] examined the dynamics of an infinitely long bar and identified features not present in the classical theory, such as wavelike oscillations spreading out from the loading region. The dynamics of peridynamic infinite bars of homogeneous and heterogeneous media have also been studied by, for example, Weckner and Abeyaratne [5] and Mikata [6].

Bobaru et al. [3] numerically investigated three kinds of displacement convergence for a given type of bar loading, with different numbers of material points and horizon sizes. The authors also studied adaptive grid refinement away from the bar ends to facilitate multiscale peridynamic modeling. They found the relative error in relation to the analytical solution of deformation decreased as the number of material points in the bar increased.

Madenci and Oterkus [7] studied the longitudinal movement of the mid-point of a peridynamic bar, constrained in one end and momentarily loaded in the other and discretized into 1000 points. The purpose of the study was to provide a benchmark example to be used in subsequent $2 \mathrm{D}$ and $3 \mathrm{D}$ modeling.

Seleson and Littlewood [8] utilized polynomial influence functions (similar to kernels) to modulate the strength of nonlocal interactions of a peristatic bar, of 100 points, with displacement constraints at the bar ends. The numerical error of displacements was reduced by half by employing the influence functions. Chen et al. [9] achieved significant improvements in the convergence of finite peridynamic bars with the corresponding classical solutions by modifying the kernel.

Aguiar et al. [10] investigated the behavior of the displacement field near the ends of a peridynamic bar and found that depending on the micromodulus, the displacement field could become unbounded near the bar ends.

Nishawala and Ostoja-Starzewski [11] suggested an inverse approach to the analytical linear elastic deformation of $1 \mathrm{D}$ bars, by first assuming a given deformation and then determining what loading was required to obtain the given deformation, resulting in polynomial functions for element-wise applied body forces.

In view of 2D, Le and Bobaru [12] compared eight methods for surface correction suggested by various authors. The maximum displacement difference between the peridynamic solutions with surface correction and the classical solutions varied from single to double digits in percentage. In general, these eight methods assume a continuum and depend on geometrical calculations to compensate for the truncated nonlocal neighborhood.

Prakash [13] used a least squares approach to calibrate on a bond level in 3D. The study obtained exact continuum mechanics behavior for material points next to a surface. However, the improvements at edges and corners were smaller than for other existing methods, 
as seen in Le and Bobaru [12]. Even though the method can be applied in 1D, the direct calibration of the equilibrium equations presented in the present work is simpler and more straightforward.

The paper is set up as follows. The next section briefly introduces peridynamic theory and considers the derivation of the effective micromodulus $c$ for a bar with a finite number of points inside the horizon. We then derive the parameters necessary to adjust bond stiffness near the ends of the 1D bar. We show that the values and the number of stiffness correction parameters are dependent on the extent of a point's neighborhood. We also derive the adjustments of the related stiffness matrix to facilitate the easy numerical implementation in peristatic/dynamic modeling, for both the stiffness matrix formulation and the most common implementation in practice, looping over equilibrium equations formulation. In the final section, the results are presented and discussed.

\section{Bond-based 1D Peridynamics}

The peridynamic equation of motion of a material point at position $\mathbf{x}$ at time $t$ is given as $[1,2]$

$$
\rho(\mathbf{x}) \ddot{\mathbf{u}}(\mathbf{x}, t)=\int_{\mathcal{H}_{\mathbf{x}}} \mathbf{f}\left(\mathbf{u}\left(\mathbf{x}^{\prime}, t\right)-\mathbf{u}(\mathbf{x}, t), \mathbf{x}^{\prime}-\mathbf{x}\right) \mathrm{d} V_{\mathbf{x}^{\prime}}+\mathbf{b}(\mathbf{x}, t) \quad \forall \mathbf{x} \in \Omega
$$

where $\Omega$ is the domain of the body, $\mathbf{u}$ is the displacement vector field, $\rho$ is the mass density, and $\mathbf{b}$ is a prescribed body force field. $\mathbf{f}$ is the pairwise force function (a vector) per unit volume squared, denoting the force exerted by the material point at $\mathbf{x}^{\prime}$ on the material point at $\mathbf{x}$. This interaction between pairs of material points is called bond, or spring in the case of a linear elastic material. The integral is defined over a region $\mathcal{H}_{\mathbf{x}}$, of radius $\delta$ called the horizon (Fig. 1). $\mathcal{H}_{\mathbf{x}}$ can be seen as a sphere, disk, or interval, for 3D, 2D, and 1D models, respectively. The material points inside the horizon, except $\mathbf{x}$ itself, are called family members of $\mathbf{x}$.

After a horizon size has been chosen, the material body is discretized by choosing a relative grid density factor $m=\delta / \Delta$, where $m$ is the number of point spacings within the horizon "radius," or size, and $\Delta$ is the constant material point spacing. Convergence studies may be performed to justify the selection of horizon and discretization. In 1D, the number of points inside the region $\mathcal{H}_{\mathbf{x}}$ is given by $N=1+2 m$. For $2 \mathrm{D}$ and $3 \mathrm{D}$ problems, the relative grid density factor $m$ should have a ratio of at least 3 [2,7] and, in many cases, 4 or higher [14-16] to provide grid independent crack growth patterns.

Fig. 1 Deformation of a 1D bar comprising seven material points, in an undeformed (bottom) and deformed (top) state. In this case, the bar end elements have the same volume, or weight, as the other five elements

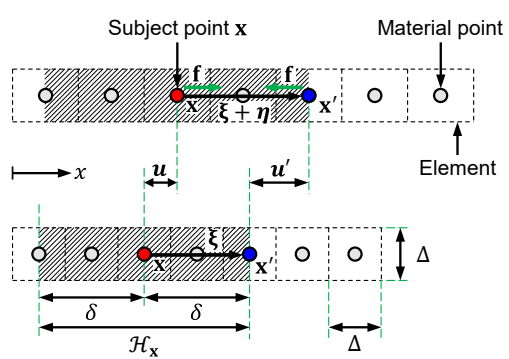


A material is called microelastic if the pairwise force $\mathbf{f}$ between material points is derivable from a micropotential $\omega[1]$ :

$$
\mathbf{f}(\boldsymbol{\eta}, \boldsymbol{\xi})=\frac{\partial \omega(\boldsymbol{\eta}, \boldsymbol{\xi})}{\partial \boldsymbol{\eta}}
$$

where $\boldsymbol{\xi}=\mathbf{x}^{\prime}-\mathbf{x}$ is the relative position of two material points in the reference configuration, and $\eta=\mathbf{u}\left(\mathbf{x}^{\prime}, t\right)-\mathbf{u}(\mathbf{x}, t)=\mathbf{u}^{\prime}-\mathbf{u}$ is the corresponding relative displacement in the deformed configuration. A linear microelastic material results in the micropotential

$$
\omega(\boldsymbol{\eta}, \boldsymbol{\xi})=\frac{c(\|\boldsymbol{\xi}\|) s^{2}\|\boldsymbol{\xi}\|}{2}
$$

where $s$ is the relative elongation of a bond:

$$
s=\frac{\|\xi+\eta\|-\|\xi\|}{\|\xi\|}
$$

Differentiation of Eq. (3) according to Eq. (2) gives

$$
\mathbf{f}(\boldsymbol{\eta}, \boldsymbol{\xi})=\frac{\partial \omega(\boldsymbol{\eta}, \boldsymbol{\xi})}{\partial \boldsymbol{\eta}}=c(\|\boldsymbol{\xi}\|) s \frac{\partial\|\boldsymbol{\xi}+\boldsymbol{\eta}\|}{\partial \boldsymbol{\eta}}=c(\|\boldsymbol{\xi}\|) s \frac{\boldsymbol{\xi}+\boldsymbol{\eta}}{\|\boldsymbol{\xi}+\boldsymbol{\eta}\|}
$$

where $(\xi+\eta) /\|\xi+\eta\|=\mathbf{e}$ is a unit vector along a line through the two end points of a bond in the deformed configuration. As we assume that a material point $\mathbf{x}$ does not interact with material points outside its horizon, $\mathbf{f}=\mathbf{0}$ for $\|\boldsymbol{\xi}\|>\delta$.

The ratio $c(\|\xi\|) /\|\xi\|$ of the particular kernel of the integrand shown in Eq. (1), is common in peridynamic mechanical problems. Other kernels are possible, and the selection influences the nonlocality, convergence, and thus the discretization applied [9].

In $1 \mathrm{D}$ axially loaded, straight bars, the vectors $\eta$ and $\xi$ are parallel, thus implying the force expression

$$
\mathbf{f}(\boldsymbol{\eta}, \boldsymbol{\xi})=c(\|\xi\|) \frac{\eta_{i}}{\|\xi\|} \frac{\xi}{\|\xi\|}
$$

where $\eta_{i}$ denotes the component of the relative displacement in the direction of the undeformed bond and $\frac{\xi}{\|\xi\|}=$ e. Note that Eq. (5a) is the linearized form of Eq. (5).

The elastic stiffness of a bond is determined by the micromodulus function $c(\|\boldsymbol{\xi}\|)$, which is conveniently obtained by equating the peridynamic strain energy density to the classical strain energy density at a point embedded within the bulk of a material body. For $1 \mathrm{D}$ problems, assuming bonds with a constant modulus, the micromodulus is obtained for a continuous bar, i.e., for a bar with an infinite number of points inside the horizon. However, for a bar with a finite number of points inside the horizon, the micromodulus is, with one exception, theoretically dependent on the number of points within the horizon. The micromodulus for a bar with a finite number of points inside the horizon is derived as follows.

Considering first an infinite number of points inside the horizon $-\delta \leq x \leq \delta$, the peridynamic strain energy density is

$$
W_{\infty}=\frac{1}{2} \int_{\mathcal{H}_{\mathbf{x}}} \omega(\boldsymbol{\eta}, \boldsymbol{\xi}) \mathrm{d} V=\frac{1}{2} \int_{\mathcal{H}_{\mathbf{x}}} \frac{c_{\infty} s^{2} \xi}{2} \mathrm{~d} V=\frac{c_{\infty} s^{2}}{4} \int_{-\delta}^{\delta} \xi A \mathrm{~d} \xi=\frac{c_{\infty} s^{2}}{4} A \delta^{2}
$$

where $\mathrm{d} V=A \mathrm{~d} \xi$ and $A$ is the cross-section. By making $W_{\infty}$ equal to the classical uniaxial strain energy density $W_{0}=E \varepsilon^{2} / 2$, the constant $c_{\infty}=2 E /\left(A \delta^{2}\right)$ is obtained with $s=\varepsilon$ [3]. 
For a finite odd number of points $N_{h}$ inside the horizon $-\delta \leq x \leq \delta$ and with $m=$ $\left(N_{h}-1\right) / 2$, the strain energy density can be written as

$$
W=\frac{\mathrm{cs}^{2}}{4} \int_{\mathcal{H}_{\mathbf{x}}} \xi \mathrm{d} V \rightarrow W_{N_{h}}=\frac{c(m) s^{2}}{4} 2 \sum_{i=1}^{m} \xi_{i} V_{i}
$$

$W_{N_{h}}=W_{\infty}$ implies that $\sum_{i=1}^{m} \xi_{i} V_{i}=A \delta^{2} / 2$.

Let $V=A \Delta$ be the volume or the weight of a bulk element. To allow any weight of a horizon element, its weight is written $V_{\delta}=(1 / 2+\alpha) A \Delta$, where $-1 / 2 \leq \alpha \leq 1 / 2$ is a dimensionless parameter to be determined.

The substitution of $V$ into the summation of Eq. (7) yields

$$
\begin{aligned}
\sum_{i=1}^{m} \xi_{i} V_{i} & =\sum_{i=1}^{m-1} i \Delta V+m \Delta(1 / 2+\alpha) A \Delta=\left[\sum_{i=1}^{m-1} i+m \Delta(1 / 2+\alpha)\right] A \Delta^{2} \\
& =\left[\frac{(m-1) m}{2}+m(1 / 2+\alpha)\right] A \Delta^{2}
\end{aligned}
$$

where the identity $\sum_{i=1}^{m} i=(m+1) m / 2$ is used.

To allow the horizon to be drawn anywhere through a horizon element, we write $\delta=$ $(m+\beta) \Delta$, where $-1 / 2 \leq \beta \leq 1 / 2$ is another dimensionless parameter to be determined. Substitution into Eq. (8) yields

$$
\sum_{i=1}^{m} \xi_{i} V_{i}=\left[\frac{(m-1) m}{2}+m(1 / 2+\alpha)\right] \frac{A \delta^{2}}{(m+\beta)^{2}}=\frac{1+2 \frac{\alpha}{m}}{\left(1+\frac{\beta}{m}\right)^{2}} \frac{A \delta^{2}}{2}
$$

Clearly, the factor $\left(1+{ }^{2 \alpha} / m\right) /(1+\beta / m)^{2}=1$ only if $\alpha=\beta=0$ (or when $m \rightarrow \infty$ ). Thus, $c$ is only independent of the number of material points within the horizon and equal to $c_{\infty}$ if the weight of the horizon element is halved, and the horizon is drawn through the middle of an end element. This is also easily verified by substituting $\delta=m \Delta$ and $V_{\delta}=V / 2$ into Eq. (9), resulting in $\sum_{i=1}^{m} \xi_{i} V_{i}=A \delta^{2} / 2$.

If the horizon is drawn half-way between two elements, and the horizon elements are given full weight, the micromodulus $c$ becomes dependent on the number of material points within the horizon. In this case, $\delta=(m+1 / 2) \Delta$ and

$$
\sum_{i=1}^{m} \xi_{i} V_{i}=\frac{1}{2}\left(m^{2}+m\right) \Delta V=\frac{1}{2}\left(m^{2}+m\right) A \Delta^{2}=\frac{m^{2}+m}{(m+1 / 2)^{2}} \frac{A \delta^{2}}{2}
$$

from which we obtain

$$
W_{N_{h}}=\frac{c(m) s^{2}}{2} \frac{m^{2}+m}{(m+1 / 2)^{2}} \frac{A \delta^{2}}{2}
$$

Finally, from $W_{N_{h}}=W_{0}$, the following micromodulus is derived:

$$
c(m)=\frac{[1+1 /(2 m)]^{2}}{1+1 / m} \frac{2 E}{A \delta^{2}}
$$

where the function $f(m)=[1+1 /(2 m)]^{2} /(1+1 / m) \rightarrow 1$ as $m \rightarrow \infty$. For $N_{h}=7, f(3)=$ 1.021 , and for $N_{h}=11, f(5)=1.008$; therefore, this dependency on $m$ can be omitted in practice.

Other end element weightings and horizon locations (of which we have seen none in the literature) yield a less favorable $m$-dependence of the micromodulus. 
We conclude that the constant micromodulus is, with two exceptions, generally dependent on the number of points/elements inside the horizon. The two exceptions are (a) an infinite number of points inside the horizon and (b) a finite number of points with the horizon drawn through the middle of half-weight horizon elements.

Other types of micromoduli (e.g., linear, higher degree polynominal) are available for $1 \mathrm{D}[3], 2 \mathrm{D}[14]$, and 3D [2].

\section{Homogenization of the Peristatic Bar}

The equation of equilibrium of a material point in a 1D-linearized peristatic bar, obtained from the basic peridynamic equation of motion [1], is, according to Bobaru et al. [3], in standard notation,

$$
\sum_{p=1}^{n} c\left(\left|x_{p}-x_{i}\right|\right) \frac{u_{p}-u_{i}}{\left|x_{p}-x_{i}\right|} V_{i p}+b_{i}=0
$$

where $u_{i}$ is the only component of the displacement vector $\mathbf{u}$, of the material point situated at $x_{i}$.

For present purposes, we note that, in particular, the micromodulus $c$, related to the bond between two (material) points, situated at $x_{p}$ and $x_{i}$, may vary from bond to bond.

A 1D peristatic bar has certain characteristic properties. In a bar loaded in uniaxial tension, by equal and opposite forces, applied at the end points of the bar, the strain in any bond between neighbor points is constant by definition, ${ }^{1}$ but varies in general piecewise, from bond to bond and along the bar. Moreover, the bond strains vary symmetrically around the mid-point/s of the bar (with an odd and even number of points), with a maximum between an end point and its nearest neighbor. The total strain of the bar varies with the number of points in the bar and the horizon size; an example is shown in Fig. 3a. The strain variations within the bar decrease slowly with an increasing number of points, and, as a result, the total strain asymptotically approaches a limit value, dependent on the horizon size. The strain variation characteristics are an important feature when modeling phenomena with a length scale of the order of point spacing but may be a nuisance when modeling phenomena of greater scales. In general, a great number of points, in the order of 100-1000 are required to model a reasonably homogeneous bar.

The variation of strain along the bar is due to different sets of bonds in the first few and last point interspaces of the bar (these differ from the constant set of bonds in the bulk of the bar). The number of end interspaces, each with a particular set of bonds, increases with the horizon size of the bar. Because the sets of bonds in the end interspaces differ, their stiffness also differ.

The idea of homogenization is simply to adjust the micromodulus (or stiffness) of certain bonds in the end interspaces, so that the same interspace stiffness is obtained in the end interspaces as in the central part of the bar. The choice of bonds for which the stiffness is to be adjusted is not arbitrary, but follows a specific procedure.

To describe our procedure, we start with the simplest case possible, namely a bar with a minimum horizon size and minimum number of points, so that the entire bar is just covered by the horizon. The end elements of the bar are full-weight elements. We then generalize the procedure to include greater horizons, bars with an arbitrary, including infinite, number of points, and bars with half-weight end elements.

\footnotetext{
${ }^{1} \mathrm{~A}$ discussion of bonds with a varying micromodulus is beyond the scope of the present article.
} 


\subsection{Introductory Example}

Writing out the first two equilibrium equations explicitly (Eq. 13) for a bar with a horizon size $\delta=2 \Delta$ and number of points $N=5$, for the first subject point, we get

$$
u_{2}-u_{1}+\frac{u_{3}-u_{1}}{2 \cdot 2}=-\frac{b_{1}}{c A}
$$

where $A=V_{\text {ip }} / \Delta$, and $\left(1^{\prime}\right)$ indicates equilibrium equation 1 , corresponding to subject point 1 . The two factors $1 / 2$ in the third term of the equation appear (a) because of the distance of two point spacings between points 1 and 3 and (b) because point 3 is on the horizon. For the second subject point, we obtain

$$
u_{1}-u_{2}+u_{3}-u_{2}+\frac{u_{4}-u_{2}}{2 \cdot 2}=-\frac{b_{2}}{c A}
$$

and so on, until subject point $N$, with the equilibrium equation number kept equal to the subject point number.

For illustration, as well as computation, the equilibrium equations are conveniently collected on the matrix-vector form $\mathbf{S u}=-\mathbf{b}$.

In standard arrangement, that is, with row number equal to equation number and column number equal to subject point number and with $\mathbf{S u}=-4 \mathbf{b} / c A$ in the present case, we get

$$
\left[\begin{array}{ccccc}
-5 & 4 & 1 & & \\
4 & -9 & 4 & 1 & \\
1 & 4 & -10 & 4 & 1 \\
& 1 & 4 & -9 & 4 \\
& & 1 & 4 & -5
\end{array}\right]\left[\begin{array}{l}
u_{1} \\
u_{2} \\
u_{3} \\
u_{4} \\
u_{5}
\end{array}\right]=-\frac{4}{A c}\left[\begin{array}{c}
b_{1} \\
0 \\
0 \\
0 \\
b_{5}
\end{array}\right]
$$

Each diagonal element in $\mathbf{S}$ is the displacement coefficient of the subject point in the corresponding equilibrium equation. The coefficients of the subject point neighbors (points within the subject point's horizon or the family of the subject point) are off-diagonal elements in the same row, taken in order.

We note in passing that for a bar with any greater number of points, the third row (in this case) is simply repeated diagonally downwards, and the last two rows, which are "double reflections" of the two first rows, are shifted column-wise accordingly.

Note that the matrix $\mathbf{S}$ for a bar with any number of points can be constructed from the $3 \times 3$ unique submatrix $\mathbf{S}_{\mathbf{u}}$, taken from the upper left corner of $\mathbf{S}$ and written as

$$
\left[\begin{array}{ccc}
-5 & 4 & 1 \\
& -9 & 4 \\
& & -10
\end{array}\right]
$$

This is symmetric for a bar with full-weight end points only.

Generally, any stiffness matrix can be constructed from its $(m+1) \times(m+1)$ unique submatrix. $^{2}$

For a bar where $m=2$, direct inspection of the bar reveals the bond sets are different only in the first and last point interspaces of the bar (but they are equal between themselves). Accordingly, we assume an adjusted bond stiffness only of the bond between points 1 and

\footnotetext{
${ }^{2}$ An even simpler but less transparent form, from which $\mathbf{S}$ can be constructed is presented in Sect. 3.5. For better transparency, the form of Eq. (17) is used in this section.
} 
2 (but not of the bond between points 1 and 3, as this choice would also affect the stiffness in the interspace between points 2 and 3 ) and between points 4 and 5 .

The loading of the bar is symmetric; therefore, its displacement is also symmetric with respect to the mid-point of the bar. Because of the symmetry, it suffices to consider half of the bar. Noting in particular that the bond stiffness is adjusted in just the first two equilibrium equations and that $b_{2}=0$, from Eqs $\left(1^{\prime}\right)$ and $\left(2^{\prime}\right)$ we get

$$
\begin{gathered}
\alpha_{21}\left(u_{2}-u_{1}\right)+\frac{u_{3}-u_{1}}{2 \cdot 2}=-\frac{b_{1}}{c A} \\
\alpha_{12}\left(u_{1}-u_{2}\right)+u_{3}-u_{2}+\frac{u_{4}-u_{2}}{2 \cdot 2}=0
\end{gathered}
$$

where $\alpha_{\mathrm{ij}}$ is a dimensionless stiffness adjustment parameter to be determined. In bond-based peridynamics, $\alpha_{21}=\alpha_{12}=\alpha$. The parameter $\alpha$ is the only unknown in Eqs $\left(1^{\prime \prime}\right)$ and $\left(2^{\prime \prime}\right)$. In particular, the simplification of Eq. $\left(2^{\prime \prime}\right)$ yields

$$
4 \alpha u_{1}-(4 \alpha+5) u_{2}+4 u_{3}+u_{4}=0
$$

Because of the abovementioned symmetry with respect to the mid-point of the bar, the displacement $u_{3}=0$. To homogenize the deformation of the bar, we further require that

$$
u_{1}=2 u_{2} \text { and } u_{4}=-u_{2}
$$

That is, point 1 is displaced twice as far as point 2 from the mid-point of the bar.

Inserting Eqs. (21a-b) in Eq. (20) and solving for $\alpha$, we get

$$
\alpha=3 / 2
$$

From Eqs. (1") and (2"), the upper left part of the submatrix $\mathbf{S}_{\mathbf{u}}$ becomes

$$
\left[\begin{array}{cc}
-(4 \alpha+1) & 4 \alpha \\
& -(4 \alpha+5)
\end{array}\right]
$$

and is symmetric. When $\alpha$ is substituted, this provides the elements of the upper left corner of $\mathbf{S}$ :

$$
\left[\begin{array}{cc}
-7 & 6 \\
& -11
\end{array}\right]
$$

The entire stiffness matrix of the homogenized bar is now easily obtained from the resulting "upper left corner."

A useful result is that Eq. (22) holds in general for a bar with an arbitrary number of points (greater than 4). To show this, consider first a bar with an odd number of points $N \geq 7$. Let $n$ be the number of points between the mid-point of the bar and the $m+1$ outermost points at a bar end (on either side of the mid-point). That is, in this case, $N=1+2(n+3)$. Then, for the mid-point,

$$
u_{n+4}=0
$$

Homogenizing requires

$$
u_{1}=(n+3) u_{n+3}, \quad u_{2}=(n+2) u_{n+3}, \quad u_{3}=(n+1) u_{n+3} \quad \text { and } u_{4}=n u_{n+3} \quad(26 \mathrm{a}-\mathrm{d})
$$

Inserting into Eq. (20) and canceling the common factor $u_{n+3}$ yields

$$
4 \alpha(n+3)-(4 \alpha+5)(n+2)+4(n+1)+n=0
$$

The factors of $n$ sum up to zero, and the remaining terms give the same solution as above

$$
\alpha=3 / 2
$$

It is left to the reader to verify that this result holds for a bar with an even number of points. 


\subsection{Second Example}

Next, we consider a bar with $\delta=3 \Delta$ and $N=7$ points. In this case, the first three equilibrium equations are of interest:

$$
\begin{gathered}
u_{2}-u_{1}+\frac{u_{3}-u_{1}}{2}+\frac{u_{4}-u_{1}}{3 \cdot 2}=-\frac{b_{1}}{c A} \\
u_{1}-u_{2}+u_{3}-u_{2}+\frac{u_{4}-u_{2}}{2}+\frac{u_{5}-u_{2}}{3 \cdot 2}=-\frac{b_{2}}{c A} \\
\frac{u_{1}-u_{3}}{2}+u_{2}-u_{3}+u_{4}-u_{3}+\frac{u_{5}-u_{3}}{2}+\frac{u_{6}-u_{3}}{3 \cdot 2}=-\frac{b_{3}}{c A} \\
\text { etc. }
\end{gathered}
$$

For a bar where $m=3$, the bond sets in the first two and the last two bar interspaces are different. In particular, the bond set in the first interspace is different from that in the second. The same holds for the last two interspaces.

Assume therefore, in view of the first example, a bond stiffness $\alpha c$ between points 1 and 2 and $\beta c$ between points 1 and 3 (and likewise at the other bar end). Noting that $b_{3}=b_{2}=0$, from $\left(1^{\prime}\right),\left(2^{\prime}\right)$ and $\left(3^{\prime}\right)$, we get respectively,

$$
\begin{gathered}
\alpha\left(u_{2}-u_{1}\right)+\frac{\beta\left(u_{3}-u_{1}\right)}{2}+\frac{u_{4}-u_{1}}{3 \cdot 2}=-\frac{b_{1}}{c A} \\
\alpha\left(u_{1}-u_{2}\right)+u_{3}-u_{2}+\frac{u_{4}-u_{2}}{2}+\frac{u_{5}-u_{2}}{3 \cdot 2}=0 \\
\frac{\beta\left(u_{1}-u_{3}\right)}{2}+u_{2}-u_{3}+u_{4}-u_{3}+\frac{u_{5}-u_{3}}{2}+\frac{u_{6}-u_{3}}{3 \cdot 2}=0 \\
\text { etc. }
\end{gathered}
$$

As before, $\alpha$ and $\beta$ do not appear in any of the remaining equations of concern. Note that there is a single unknown in each of the equations $\left(2^{\prime \prime}\right)$ and $\left(3^{\prime \prime}\right)$, with a vanishing right-hand side.

The equations of interest, $\left(2^{\prime \prime}\right)$ and $\left(3^{\prime \prime}\right)$, can be simplified to

$$
\begin{gathered}
6 \alpha u_{1}-(6 \alpha+10) u_{2}+6 u_{3}+3 u_{4}+u_{5}=0 \\
3 \beta u_{1}+6 u_{2}-(3 \beta+16) u_{3}+6 u_{4}+3 u_{5}+u_{6}=0
\end{gathered}
$$

At the mid-point of the bar $u_{4}=0$. To homogenize the bar, we require that

$$
u_{1}=3 u_{3}, \quad u_{2}=2 u_{3}, \quad u_{5}=-u_{3} \quad \text { and } \quad u_{6}=-u_{2}
$$

Inserting Eqs. (37a-d) in ( $\left.2^{\prime \prime \prime}\right)$ and ( $\left.3^{\prime \prime \prime}\right)$ and solving for $\alpha$ and $\beta$, we get

$$
\alpha=5 / 2 \text { and } \beta=3 / 2
$$

As above, these parameters apply to a bar with any greater number of points, odd or even. With $\mathbf{S u}=-6 \mathbf{b} /(c A)$ in this case, the submatrix $\mathbf{S}_{\mathbf{u}}$ becomes

$$
\left[\begin{array}{cccc}
-10 & 6 & 3 & 1 \\
& -16 & 6 & 3 \\
& & -19 & 6 \\
& & & -20
\end{array}\right]
$$


From Eqs. $\left(1^{\prime \prime}\right),\left(2^{\prime \prime}\right)$ and $\left(3^{\prime \prime}\right)$, for the upper left part of the submatrix $\mathbf{S}_{\mathbf{u}}$ we obtain

$$
\left[\begin{array}{ccc}
-(6 \alpha+3 \beta+1) & 6 \alpha & 3 \beta \\
& -(6 \alpha+10) & 6 \\
& & -(3 \beta+16)
\end{array}\right]
$$

which, when $\alpha$ and $\beta$ are inserted, provides the upper left corner of $\mathbf{S}$,

$$
\frac{1}{2}\left[\begin{array}{ccc}
-41 & 30 & 9 \\
& -50 & 12 \\
& & -41
\end{array}\right]
$$

\subsection{Third Example}

Finally, we consider a bar with $\delta=4 \Delta$ and $N=9$ points. The equilibrium equations of interest in this case are

$$
\begin{gathered}
u_{2}-u_{1}+\frac{u_{3}-u_{1}}{2}+\frac{u_{4}-u_{1}}{3}+\frac{u_{5}-u_{1}}{4 \cdot 2}=-\frac{b_{1}}{c A} \\
u_{1}-u_{2}+u_{3}-u_{2}+\frac{u_{4}-u_{2}}{2}+\frac{u_{5}-u_{2}}{3}+\frac{u_{6}-u_{2}}{4 \cdot 2}=-\frac{b_{2}}{c A} \\
\frac{u_{1}-u_{3}}{2}+u_{2}-u_{3}+u_{4}-u_{3}+\frac{u_{5}-u_{3}}{2}+\frac{u_{6}-u_{3}}{3}+\frac{u_{7}-u_{3}}{4 \cdot 2}=-\frac{b_{3}}{c A} \\
\frac{u_{1}-u_{4}}{3}+\frac{u_{2}-u_{4}}{2}+u_{3}-u_{4}+u_{5}-u_{4}+\frac{u_{6}-u_{4}}{2}+\frac{u_{7}-u_{4}}{3}+\frac{u_{8}-u_{4}}{4 \cdot 2}=-\frac{b_{4}}{c A}
\end{gathered}
$$

etc.

For a bar where $m=4$, the bond sets in the three first and last bar interspaces are different.

Assume, therefore, in this case, bond stiffness $\alpha c$ between points 1 and 2, $\beta c$ between points 1 and 3 , and $\gamma c$ between points 1 and 4 (and likewise at the other bar end). Noting that $b_{4}=b_{3}=b_{2}=0$, from $\left(1^{\prime}\right),\left(2^{\prime}\right),\left(3^{\prime}\right)$, and $\left(4^{\prime}\right)$ we get, respectively,

$$
\begin{gathered}
\alpha\left(u_{2}-u_{1}\right)+\frac{\beta\left(u_{3}-u_{1}\right)}{2}+\frac{\gamma\left(u_{4}-u_{1}\right)}{3}+\frac{u_{5}-u_{1}}{4 \cdot 2}=-\frac{b_{1}}{c A} \\
\alpha\left(u_{1}-u_{2}\right)+u_{3}-u_{2}+\frac{u_{4}-u_{2}}{2}+\frac{u_{5}-u_{2}}{3}+\frac{u_{6}-u_{2}}{4 \cdot 2}=0 \\
\frac{\beta\left(u_{1}-u_{3}\right)}{2}+u_{2}-u_{3}+u_{4}-u_{3}+\frac{u_{5}-u_{3}}{2}+\frac{u_{6}-u_{3}}{3}+\frac{u_{7}-u_{3}}{4 \cdot 2}=0 \\
\frac{\gamma\left(u_{1}-u_{4}\right)}{3}+\frac{u_{2}-u_{4}}{2}+u_{3}-u_{4}+u_{5}-u_{4}+\frac{u_{6}-u_{4}}{2}+\frac{u_{7}-u_{4}}{3}+\frac{u_{8}-u_{4}}{4 \cdot 2}=0
\end{gathered}
$$

As before, $\alpha, \beta$, and $\gamma$ do not appear in any of the remaining equilibrium equations. The equations with a single unknown each, Eqs. $\left(2^{\prime \prime}\right),\left(3^{\prime \prime}\right)$, and $\left(4^{\prime \prime}\right)$, can be simplified to

$$
\begin{gathered}
24 \alpha u_{1}-(24 \alpha+47) u_{2}+24 u_{3}+12 u_{4}+8 u_{5}+3 u_{6}=0 \\
12 \beta u_{1}+24 u_{2}-(12 \beta+71) u_{3}+24 u_{4}+12 u_{5}+8 u_{6}+3 u_{7}=0 \\
8 \gamma u_{1}+12 u_{2}+24 u_{3}-(8 \gamma+83) u_{4}+24 u_{5}+12 u_{6}+8 u_{7}+3 u_{8}=0
\end{gathered}
$$

At the mid-point of the bar, $u_{5}=0$. To homogenize the bar, we require that

$$
u_{1}=4 u_{4}, \quad u_{2}=3 u_{4}, \quad u_{3}=2 u_{4}, \quad u_{6}=-u_{4}, \quad u_{7}=-u_{3} \quad \text { and } \quad u_{8}=-u_{2}
$$

Inserting Eq. (53a-f) into ( $\left.2^{\prime \prime \prime}\right),\left(3^{\prime \prime \prime}\right)$, and ( $\left.4^{\prime \prime \prime}\right)$ and solving for $\alpha, \beta$ and $\gamma$, results in

$$
\alpha=7 / 2, \quad \beta=5 / 2 \text { and } \gamma=3 / 2
$$


Again, these parameters apply to a bar with any greater number of points, odd or even.

When $\mathbf{S u}=-24 \mathbf{b} /(c A)$, the submatrix $\mathbf{S}_{\mathbf{u}}$ becomes

$$
\left[\begin{array}{ccccc}
-47 & 24 & 12 & 8 & 3 \\
& -71 & 24 & 12 & 8 \\
& & -83 & 24 & 12 \\
& & & -91 & 24 \\
& & & & -94
\end{array}\right]
$$

From $\left(1^{\prime \prime}\right),\left(2^{\prime \prime}\right),\left(3^{\prime \prime}\right)$, and $\left(4^{\prime \prime}\right)$, for the upper left part of the submatrix $\mathbf{S}_{\mathbf{u}}$, we obtain

$$
\left[\begin{array}{cccc}
-(24 \alpha+12 \beta+8 \gamma+3) & 24 \alpha & 12 \beta & 8 \gamma \\
& -(24 \alpha+47) & 24 & 12 \\
& & -(12 \beta+71) & 24 \\
& & & -(8 \gamma+83)
\end{array}\right]
$$

which, when $\alpha, \beta$, and $\gamma$ are inserted, results in the upper left corner of $\mathbf{S}_{\mathbf{u}}$

$$
\left[\begin{array}{cccc}
-129 & 84 & 30 & 12 \\
& -131 & 24 & 12 \\
& & -101 & 24 \\
& & & -95
\end{array}\right]
$$

\subsection{Generalization}

From the three examples, we conclude that for a bar with horizon $m$ (point spacings), the number of solving parameters required for homogenization is $m-1$, and the solving parameters for any $m$ form the set

$$
\alpha_{i}=m-i+1 / 2, \quad i=1,2, \ldots m-1
$$

In certain equilibrium equations, the product $\alpha V_{\text {ip }}$ occurs (before simplification). For a bar with half-weight end points, this product can be read as $(2 \alpha) \frac{V_{i p}}{2}$, where $V_{\text {ip }} / 2$ is the weight of a half-weight end point. Inspection of the relevant equilibrium equation makes it clear that $2 \alpha$ can be seen as the resulting stiffness coefficient of the corresponding bond. Thus, the homogenizing parameters for a bar with half-weight end points are

$$
\alpha_{i}=2(m-1)+1, \quad i=1,2, \ldots m-1
$$

The homogenizing parameters for a bar with half-weight end points are given in Appendix A.

\subsection{Stiffness Matrix}

As stated above, the equilibrium equations of the points/elements of a 1D bar with $N$ elements are conveniently written in matrix form $\mathbf{S u}=\mathbf{b}$, where $\mathbf{S}$ is an $N \times N$ stiffness matrix. This formulation allows the solution of a problem to be written formally as $\mathbf{u}=$ $\mathbf{S}^{-1} \mathbf{b}$, or in Matlab code $\mathbf{u}=\mathbf{S} \backslash \mathbf{b}$.

In terms of conventional equation and point numbering, $\mathbf{S}$ takes a characteristic diagonal structure.

For a bar with horizon $m$, the first $m$ rows are unique, and the last $m$ rows are "double reflections" of the first $m$ rows. The rows between show a repetitive pattern diagonally. The first element of row $m+1$ is situated in column one, and the last non-zero element in this row appears in column $2 m+1$. For $N=2 m+1$, however, all elements of row $m+1$ are 
non-zero. The set of non-zero elements of row $m+1$ is simply displaced one column to the right for every row-wise step downwards to row $N-m$.

\subsubsection{Full-weight Bar End Elements}

In the case of a bar with $m=4, N=9$ and full-weight end points, i.e.,the left-hand side of the equilibrium equation of element 5 , the mid-element, is

$$
\frac{u_{1}-u_{5}}{2 \cdot 4}+\frac{u_{2}-u_{5}}{3}+\frac{u_{3}-u_{5}}{2}+u_{4}-u_{5}+u_{6}-u_{5}+\frac{u_{7}-u_{5}}{2}+\frac{u_{8}-u_{5}}{3}+\frac{u_{9}-u_{5}}{2 \cdot 4}
$$

where the denominators 2,3 , and 4 represent the number of point spacings between point 5 and its neighbors. A factor $1 / 2$ is inserted in the first and the last term because points 1 and 9 are on the horizon. To obtain the elements of $\mathbf{S}$, the equation is rewritten as

$$
\frac{1}{2 \cdot 4} u_{1}+\frac{1}{3} u_{2}+\frac{1}{2} u_{3}+u_{4}-2\left(\frac{1}{2 \cdot 4}+\frac{1}{3}+\frac{1}{2}+1\right) u_{5}+u_{6}+\frac{1}{2} u_{7}+\frac{1}{3} u_{8}+\frac{1}{2 \cdot 4} u_{9}
$$

With $j=m+1$, the first repetitive row is obtained as

$$
\begin{aligned}
& \frac{1}{2 \cdot 4} u_{j-4}+\frac{1}{3} u_{j-3}+\frac{1}{2} u_{j-2}+u_{j-1}-2\left(\frac{1}{2 \cdot 4}+\frac{1}{3}+\frac{1}{2}+1\right) u_{j} \\
& +u_{j+1}+\frac{1}{2} u_{j+2}+\frac{1}{3} u_{j+3}+\frac{1}{2 \cdot 4} u_{j+4}
\end{aligned}
$$

from which it is inferred that in the general case, the first and the last elements of the row are

$$
S(j, j-m)=S(j, j+m)=\frac{1}{2 m}
$$

and the remaining elements, except element $j$, are obtained by stepping $i$ (a) backwards from $m-1$ to 1 and (b) forwards from 1 to $m-1$ :

$$
S(j, j \mp i)=\frac{1}{i}
$$

The terms inside the brackets in the equation can be written as $\frac{1}{2 m}+\sum_{i=1}^{m-1} \frac{1}{i}$, and thus,

$$
S(j, j)=-\left(\frac{1}{m}+2 \sum_{i=1}^{m-1} \frac{1}{i}\right)
$$

The left-hand side of the equilibrium equation of element 4 is

$$
\frac{u_{1}-u_{4}}{3}+\frac{u_{2}-u_{4}}{2}+u_{3}-u_{4}+u_{5}-u_{4}+\frac{u_{6}-u_{4}}{2}+\frac{u_{7}-u_{4}}{3}+\frac{u_{8}-u_{4}}{2 \cdot 4}
$$

Here, element 1 is within the horizon, but element 8 is on the horizon. Rewriting the equation as above gives

$$
\frac{1}{3} u_{1}+\frac{1}{2} u_{2}+u_{3}-2\left(\frac{1}{3}+\frac{1}{2}+1+\frac{1}{4 \cdot 4}\right) u_{4}+u_{5}+\frac{1}{2} u_{6}+\frac{1}{3} u_{7}+\frac{1}{2 \cdot 4} u_{8}
$$

The net effect is that copies of the coefficients in Eq. (61) are displaced one step upwards and one step backwards (left) in $\mathbf{S}$, and the outgoing coefficient of element 1 in Eq. (61) is added to the coefficient of element 4 in Eq. (67).

Going upwards, an outgoing coefficient from any equation is added to the diagonal coefficient in the next above equation (because a row represents the coefficients of the equilibrium equation of the subject point). This pattern is repeated up to and including the first 
equation. Thus, for any step upwards, we first calculate

$$
S(n, i)=S(n+1, i+1)
$$

where $i$ is the column number. Then, we adjust the diagonal element

$$
S(j, j)=S(j, j)+S(j+1,1)
$$

Going downwards, a similar repetitive pattern is mirrored in Eqs. $N-m+1$ to $N$, but outgoing coefficients are now on the right-hand side of $\mathbf{S}$. Going from any equation to the one immediately below it, the two above equations now become

$$
S(n, i)=S(n-1, i-1)
$$

and

$$
S(j, j)=S(j, j)+S(j-1, N)
$$

In summary, the stiffness matrix of a bar of any horizon size and with any number of points can be constructed by expanding the following set of $m+1$ coefficients,

$$
\frac{1}{2 m}, \frac{1}{m-1}, \ldots, \frac{1}{m-i}, \ldots, 1,-\left(\frac{1}{m}+2 \sum_{i=1}^{m-1} \frac{1}{i}\right)
$$

and applying the rules stated in Eqs. (68) to (71).

The corresponding procedure for half-weight bar end elements is described in Appendix A. The schemes are fairly easy to implement numerically.

\section{Results and Discussion}

We begin the section with an overview of some characteristic results of this work, including a summary of the deformation of the 1D bar types considered here. This is followed by results and discussion of four different cases. The first case summarizes graphically the displacement, relative error, and convergence of non-homogenized and homogenized bars (see Section 3). The second case considers the displacement, relative error, and convergence of bars with half-weight end elements (see Appendix A). The third example presents a benchmark example of a longitudinally vibrating bar. The fourth and final case examines the strain energy density as a function of position.

Both the stiffness matrix (static) and the looping over equilibrium equations (quasi-static) formulations have been applied, and the type is stated when required. The numerical implementation was done in Matlab. Static codes were in-house, and quasi-static codes, readily available in Madenci and Oterkus [7] in Fortran language, were worked upon. Numerical implementation of static solutions is described in Section 3.5.

\subsection{Overview of Non-Homogenized and Homogenized 1D Peridynamic Bars}

In this section, we compare the total strain of non-homogenized and homogenized 1D bars, with half-weight and full-weight end elements and horizon sizes 2, 3, and 4 times the element spacing, loaded in uniaxial tension, to the corresponding classical continuum mechanics strain. The displacements of a bar, from which the total strain is obtained, are calculated using the stiffness matrix static solution method.

The total strain of a peridynamic bar in uniaxial tension is, in general, a function of the number of points in the bar. The total strain is written as $\varepsilon(N)=f(N) \sigma / E$, where $N$ is 
Table 1 Dimensionless strain function $\mathrm{f}(\mathrm{N})$

\begin{tabular}{llllll}
\hline$\delta$ & Half-weight bar end elements & & & Full-weight bar end elements \\
\cline { 2 - 3 } \cline { 5 - 6 } & Non-homogenized & Homogenized & & Non-homogenized & Homogenized \\
\hline $2 \Delta$ & $f(N) \approx \frac{7+5 N}{4(N-1)}$ & $f(N)=8 / 7$ & & $f(N) \approx \frac{1}{N}\left(\frac{15}{17}+N\right)$ & $f(N)=1$ \\
& $f(5)=2, f(\infty)=5 / 4$ & & $f(5)=20 / 17, f(\infty)=1$ & $f(N)=1$ \\
$3 \Delta$ & $f(N) \approx \frac{2583+495 N}{440(N-1)}$ & $f(N)=18 / 17$ & $f(N) \approx \frac{1}{N}\left(\frac{1841}{718}+N\right)$ & $f(N)=1$ \\
$4 \Delta$ & $f(7)=126 / 55, f(\infty)=9 / 8$ & & $f(7)=981 / 718, f(\infty)=1$ & \\
\hline
\end{tabular}

Integers and fractions are exact analytically; decimal numbers are numerical approximations

the number of points in the bar, $f(N)$ is a dimensionless function, and $\varepsilon=\sigma / E$ is the corresponding strain in a classical continuum mechanics bar.

The derived analytical expressions and numerical results of the function $f(N)$ are summarized in Table 1 for non-homogeneous and homogeneous bars, half-weight and full-weight bar end elements, and $\delta=2 \Delta$ to $4 \Delta$.

The values of the function $f(N)$, given as integers or fractions, are exact analytical values, while decimal numbers are numerical approximations. The exact analytical values of $f(N)$ are increasingly complex ratios of the products of prime numbers with increasing $N$ and $m$. Already for $m=4$ and $N=9$, this ratio involves 10-digit numbers, and is therefore considered of little interest in practice; accordingly, we give only the numerical approximation. For $m=2$ and 3, it is possible to find surprisingly simple approximation formulae, through curve fitting (against numerical approximations), with an error generally smaller than $1 \%$. However, the end values of these approximations are exact analytical values. As the end values of $f$ for $m=4$ are numerical approximations, we have abstained from further approximation of the end values.

Note that for non-homogenized bars of both end element types, the total strain of a bar is a function of the number of points in the bar. In particular, note that for a bar with fullweight end elements, the strain converges towards the classical value with increasing $N$, independent of horizon size.

For homogenized bars of both end element types, the total strain is a constant, independent of the number of points in the bar.

Further, for a homogenized bar with half-weight elements, the total strain is a function of the horizon size.

Finally, for a homogenized bar with full-weight end elements, the total strain is independent of horizon size and exactly equal to the classical strain.

\subsection{Full-weight end Elements-Case 1}

Figures 2 and 3 show the displacement of the bar ends using the homogenization parameters derived in Section 3 for a bar with full-weight end elements. The bar is one meter in length and constrained by $u_{(N-1) / 2}=0$. Young's modulus is $E=200 \mathrm{GPa}$, and the stress $\sigma=$ $200 \mathrm{MPa}$ is applied at the bar end elements. The solution is based on a stiffness matrix static implementation in Fig. 2 and on the quasi-static implementation in Fig. 3.

The relative displacement error in Fig. $2 \mathrm{a}$ is shown in Fig. $2 \mathrm{~b}$. It is calculated as $(u-$ $\left.u_{0}\right) / u_{0}$, where $u$ and $u_{0}$ are the peridynamic and analytical displacements, respectively. The 


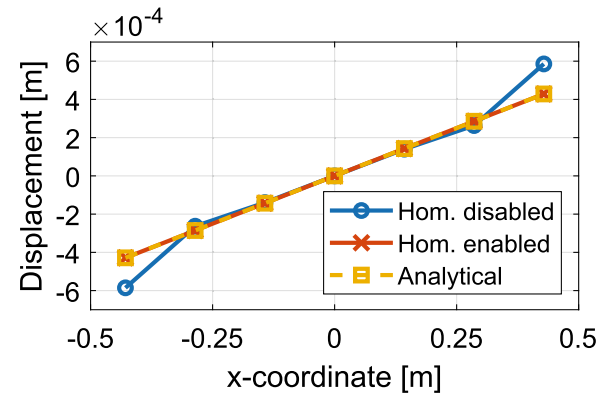

(a)

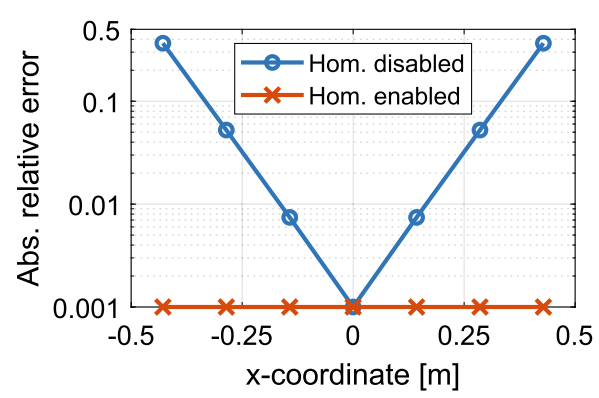

(b)

Fig. 2 Peridynamic model with $\delta=3, N=7$ and full-weight end elements, compared to the analytical solution. Homogenization (hom.) parameters are $\alpha=3 / 2$ and $\beta=5 / 2$

analytical solution of the elongation of the bar is simply $u=L \sigma / E$, so that the analytical total strain is $\varepsilon=u_{0} / L=\sigma / E$. For the analytical solution, $f(N)=1$.

Figure 3 shows the relative error as a function of the horizon $\delta$ and number of material points $N$. The relative error in $u$ for $\delta=3, N=100$ is $\varepsilon=2.2 \%$ (Fig. 3a). In Fig. 3b, the relative error increases with $N$, because the convergence of the quasi-static implementation to a steady-state solution depends on the number of time steps and material points $N . \delta=1$ is the special case of peridynamics reduced to classical continuum mechanics.

\subsection{Half-weight end Elements-Case 2}

In the second case, we study the bar with half-weight end elements (Figs. 4 and 5). Figure 5 shows that neither of the solutions approaches the exact analytical solution $f(N)=1$. However, $\delta=4 \Delta$ requires the least correction. The results are from the stiffness matrix implementation.

\subsection{Longitudinally Vibrating Bar-Case 3}

Next, we study a benchmark example in Madenci and Oterkus [7], considering the dynamics of a longitudinally vibrating bar. The bar is constrained at the left end element by $u_{1}=0$;

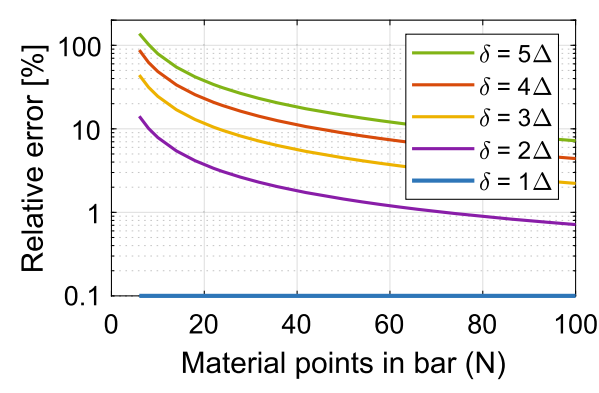

(a)

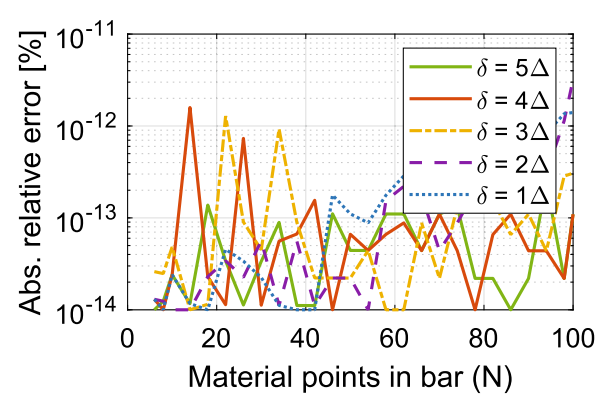

(b)

Fig. 3 Relative error in $u$ at the end element with full-weight. a Non-homogenized and b homogenized. Homogenization parameters are found using Eq. (59) 


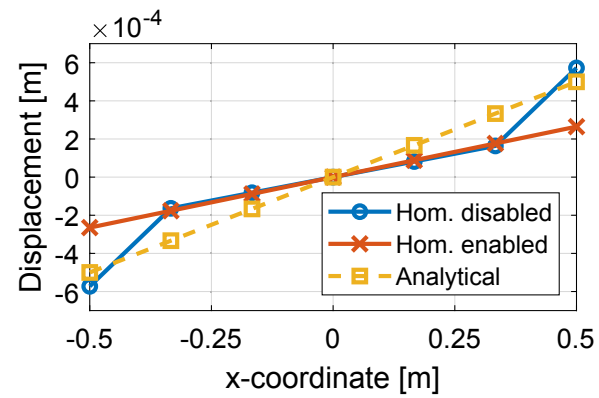

(a)

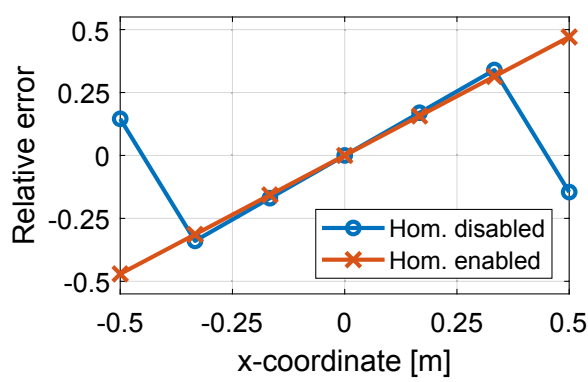

(b)

Fig. 4 Peridynamic model with $\delta=3, N=7$ and half-weight end elements, compared to the analytical solution. Homogenization (hom.) parameters are $\alpha=3$ and $\beta=5$

it has a length $L=1 \mathrm{~m}$, an elastic modulus $E=200 \mathrm{GPa}$, and is loaded by an initial strain condition of $\partial u_{0} / \partial x=0.001 H(\Delta t-t)$, where $H$ is the unit step function. The analytical solution is given by Rao [18] as

$$
u_{x}(x, t)=\frac{8 \varepsilon L}{\pi^{2}} \sum_{n=0}^{\infty} \frac{(-1)^{n}}{(2 n+1)^{2}} \sin \left(\frac{(2 n+1) \pi x}{2 L}\right) \cos \left(\sqrt{\frac{E}{\rho}} \frac{(2 n+1) \pi t}{2 L}\right)
$$

where $\varepsilon$ and $\rho$ are strain and density, respectively.

The homogenized solution shows closer agreement with the analytical solution than the non-homogenized solution in Fig. 6a-c. However, the results are affected by the extent of the horizon $\delta$, as seen in Fig. 7. The homogenized solution shows a relatively more constant error throughout $\delta=1 \Delta$ to $5 \Delta$ than the non-homogenized solution. The time step range on the $x$-axis of Fig. 7a-e varies, as the time step size depends on the horizon $\delta$. Figure $7 \mathrm{c}$ is shown in full in Fig. 8, as it may be difficult to assess how well the results in Fig. 7 resemble the analytical solution.

\subsection{Strain Energy Density as a Function of Position-Case 4}

As certain attempts to reduce boundary effects in higher dimension problems involve correction of the energy density of material points near a boundary (see [12]), it is of interest to study the resulting strain energy density of the homogenized bar.

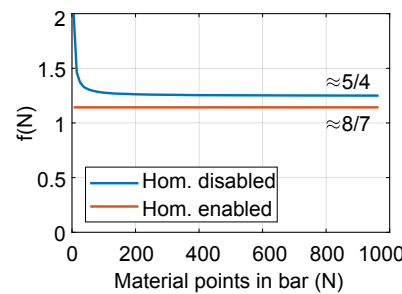

(a)

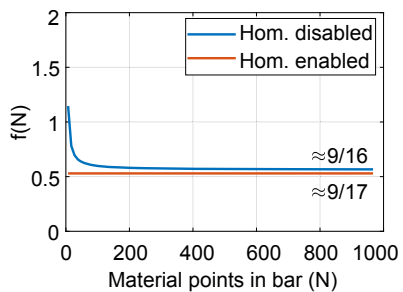

(b)

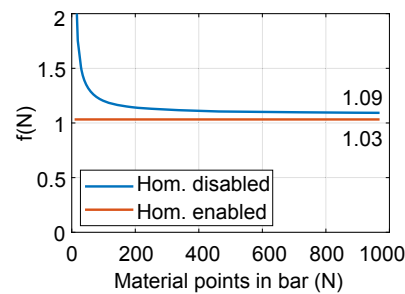

(c)

Fig. $5 f(N)$ for peridynamic model with half-weight end elements and horizon $\delta=2 \Delta$ to $\Delta 4$, (a-c), respectively 


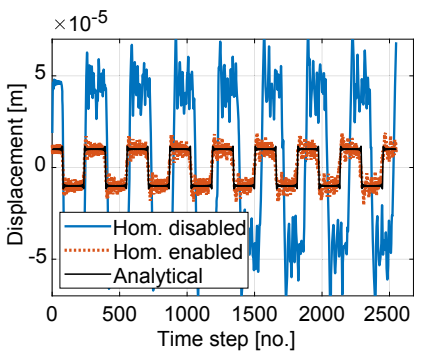

(a)

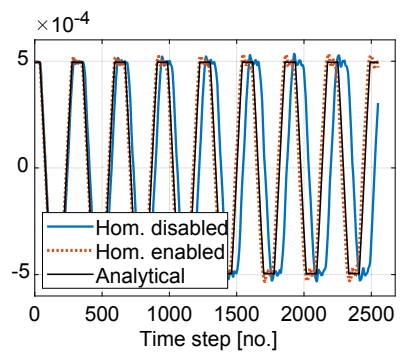

(b)

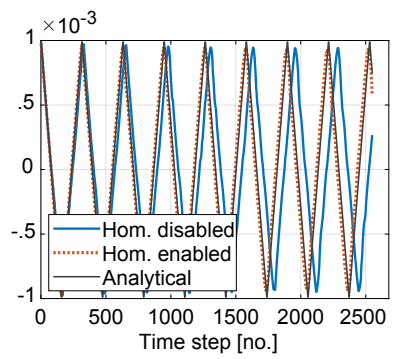

(c)

Fig. 6 Displacement of material points $\mathbf{a} i=2$, b $i=N / 2$ and $\mathbf{c} i=N . \delta=5 \Delta$ and $N=100$. Homogenization parameters are from Eq. (59)

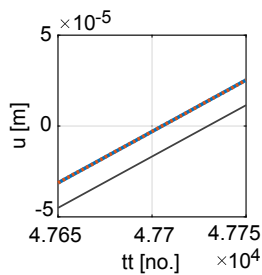

(a)

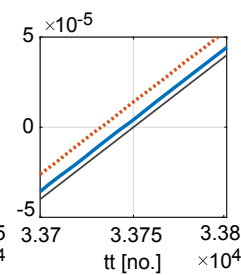

(b)

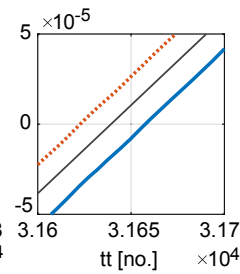

(c)

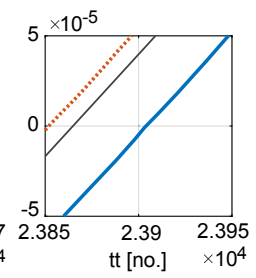

(d)

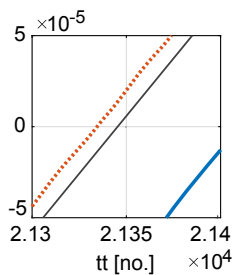

(e)

Fig. 7 Displacement of material point $i=N / 2$ zoomed in at vibration cycle seven. $N=1000$ and $\delta=1 \Delta$ to $5 \Delta$ (a-e), respectively. The line style is as per Fig. 6, and tt stands for time steps

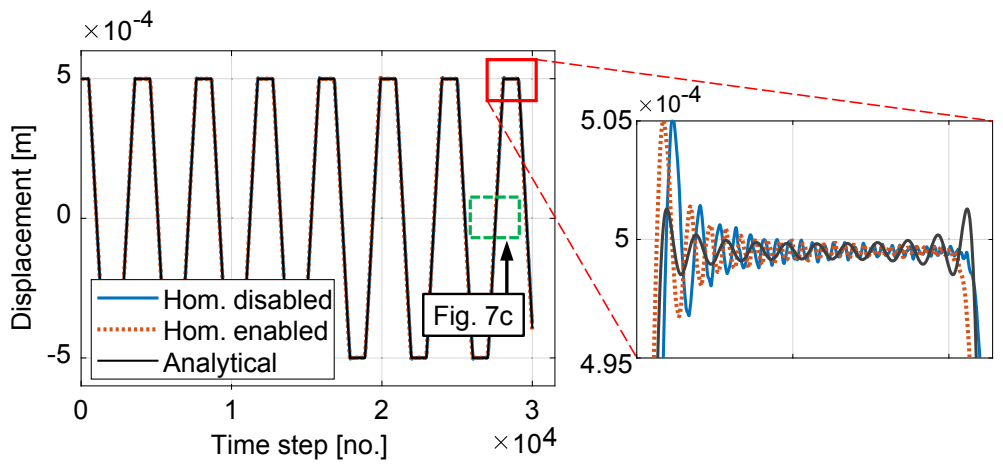

Fig. 8 Full picture of Fig. 7c 
Fig. 9 Relative values of the strain energy density $\left(\sum c \xi V\right)$

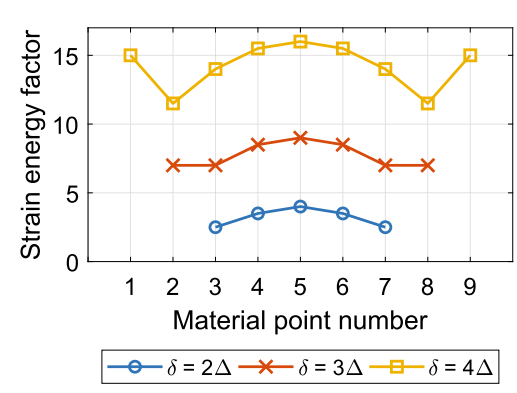

The peridynamic strain energy density is as follows:

$$
W=\frac{1}{2} \int_{\mathcal{H}_{\mathbf{x}}} \omega \mathrm{d} V_{\mathbf{x}^{\prime}}
$$

where $\omega=c s^{2} \xi / 2$. For a bar with a finite number of points inside the horizon,

$$
W \rightarrow \frac{1}{2} \sum_{\mathcal{H}_{\mathbf{x}}} \frac{c s^{2} \xi}{2} V_{i}
$$

To evaluate the strain energy per material point, it is sufficient to calculate the relative values of $W$ (instead of the absolute values). The stretch $s$ is constant by definition in the homogeneous bar. The weight and thus the volume $V$ of an element inside the horizon is 1 and on the horizon $1 / 2$. The micromodulus $c$ is corrected to achieve a homogeneous bar. Therefore, the relative values are given by $\sum c \xi V$.

A plot of $W$ against the material point numbers for horizons $2 \Delta-4 \Delta$ displays no monotonous pattern, as shown in Fig. 9. Away from the end effects, at the middle material point, note that $W \propto(\delta / \Delta)^{2} ; W_{\Delta 2}(i=3)=4, W_{\Delta 3}(i=4)=9$, and $W_{\Delta 4}(i=5)=16$. $W=4,9$, and 16 are also the relative energy densities at material points away from end effects in a long bar. The background calculations for Fig. 9 are given in Appendix B.

\subsection{Discussion}

Common types of convergences studied by, e.g., Bobaru et al. [3], Hu et al. [17] and Madenci and Oterkus [7] are $\delta$-convergence and $m$-convergence. In $\delta$-convergence, the number of material points covered by the horizon $m$ is fixed, while the horizon $\delta$ is decreased. In $m$-convergence, the horizon size is kept fixed, while the number of material points covered by the horizon $m$ is increased. The total number of material points $N$ is increased in both cases.

Bobaru et al. [3] studied the convergence of the displacement of a 1D peristatic bar, much longer than the distance between two central material points (with one further point between them) at which they applied two equal and opposite, i.e., self-balanced, forces. They considered a set of points with equal spacing and $2 m+1$ points in the region $[x-\delta, x+$ $\delta$ ] ( $\delta$ is the horizon). They then studied solution convergence by decreasing/increasing the horizon size $\delta$ and the number of points $m$ covered by the horizon. Uniform convergence to the classical solutions of static and dynamic elasticity problems in $1 \mathrm{D}$ was obtained in the limit of the horizon going to zero.

In the present study, the numerical peridynamic approximation for the homogenized bar with full-weight end elements equals the classical solution for any horizon size and admissible number of points in a bar (Fig. 3). 
It is tempting to speculate whether our presented homogenization method for 1D bars can be extended to higher dimensions. So far, a preliminary homogenization attempt with a $2 \mathrm{D}$ linearized rectangular plate with horizon two has been successful in a single case only, i.e., for uniform biaxial loading, but not for uniaxial loading or pure shear. It appears that each loading case requires a particular homogenization scheme, and no general homogenization procedure has yet been formulated.

\section{Conclusions}

The total strain of a peristatic/dynamic bar loaded in uniaxial tension strongly depends on the number of material points $N$ of the bar and on the horizon size $\delta$. In this paper, the total strain was derived analytically and computed numerically for non-homogenized and homogenized 1D bars with half-weight and full-weight end elements.

For non-homogenized bars of both end element types, the total strain of a bar was found to be a function of the number of points in the bar; in particular, for a bar with full-weight end elements, the strain converges towards the classical value with increasing $N$.

For homogenized bars of both end element types, the total strain is a constant, independent of the number of points in the bar. For a homogenized bar with half-weight end elements, the total strain is a function of the horizon size. Further, for a homogenized bar with full-weight end elements, the total strain is independent of horizon size and exactly equal to the classical strain. Finally, the deformation of a homogenized bar comes closer than that of a non-homogenized bar to the analytical solution of the longitudinally vibrating bar benchmark problem.

Acknowledgments This work has been written out of pure interest in the fascinating branch of peridynamics of solid mechanics without any particular funding. The authors are, however, grateful for the time and facilities offered by LTU, Luleå University of Technology.

Open Access This article is licensed under a Creative Commons Attribution 4.0 International License, which permits use, sharing, adaptation, distribution and reproduction in any medium or format, as long as you give appropriate credit to the original author(s) and the source, provide a link to the Creative Commons licence, and indicate if changes were made. The images or other third party material in this article are included in the article's Creative Commons licence, unless indicated otherwise in a credit line to the material. If material is not included in the article's Creative Commons licence and your intended use is not permitted by statutory regulation or exceeds the permitted use, you will need to obtain permission directly from the copyright holder. To view a copy of this licence, visit http://creativecommons.org/licenses/by/4.0/.

Funding Information Open access funding provided by Lulea University of Technology.

\section{Appendix A: Homogenizing parameters for a bar with half-weight end points}

For a bar with $\delta=2 \Delta, N=5$ points, half-weight end elements and $\mathbf{S u}=-4 \mathbf{b} /(c A)$, the stiffness submatrix $\mathbf{S}_{u}$ is

$$
\left[\begin{array}{ccc}
-5 & 4 & 1 \\
2 & -7 & 4 \\
1 & 4 & -10
\end{array}\right]
$$


Note that $\mathbf{S}_{u}$ is not symmetric.

Let the micromodulus of an end bond concerned be $\alpha c$, where $c$ is the micromodulus of the remaining ordinary bonds. From the subject point equilibrium equations, we get the elements of the upper left corner of $\mathbf{S}$ as

$$
\left[\begin{array}{cc}
-(4 \alpha+1) & 4 \alpha \\
2 \alpha & -(5+2 \alpha)
\end{array}\right]
$$

$\alpha=3$ gives

$$
\left[\begin{array}{cc}
-13 & 12 \\
6 & -11
\end{array}\right]
$$

For a bar with horizon $\delta=3 \Delta, N=7$ points and $\mathbf{S u}=-12 \mathbf{b} /(c A)$, the stiffness submatrix $\mathbf{S}_{u}$ is

$$
\left[\begin{array}{cccc}
-20 & 12 & 6 & 2 \\
6 & -26 & 12 & 6 \\
3 & 12 & -35 & 12 \\
2 & 6 & 12 & -40
\end{array}\right]
$$

Let the micromodulus of the bonds concerned in the point spacings 1-2 and $(N-1)-N$ be $\alpha c$ and in 1-3 and $(N-2)-N$ be $\beta c$. From the subject point equilibrium equations, we get the elements of the upper left corner of $\mathbf{S}$ as

$$
\left[\begin{array}{ccc}
-2(6 \alpha+3 \beta+1) & 12 \alpha & 6 \beta \\
6 \alpha & -2(3 \alpha+10) & 12 \\
3 \beta & 12 & -(3 \beta+32)
\end{array}\right]
$$

$\alpha=5$ and $\beta=3$ yield the "upper left corner"

$$
\left[\begin{array}{ccc}
-80 & 60 & 18 \\
30 & -50 & 12 \\
9 & 12 & -41
\end{array}\right]
$$

For a bar with horizon $\delta=4 \Delta, N=9$ points, and $\mathbf{S u}=-24 \mathbf{b} /(c A)$, the stiffness submatrix $\mathbf{S}_{u}$ is

$$
\left[\begin{array}{ccccc}
-47 & 24 & 12 & 8 & 3 \\
12 & -59 & 24 & 12 & 8 \\
6 & 24 & -77 & 24 & 12 \\
4 & 12 & 24 & -87 & 24 \\
3 & 8 & 12 & 24 & -94
\end{array}\right]
$$

This case requires adjustment of the stiffness of the concerned bonds in point spacings 1-2, $1-3$, and $1-4$, as well as their counterparts at the other end of the bar. 
In addition to $\alpha$ and $\beta$, let the micromodulus of the concerned bonds in point spacings $1-4$ and $(N-3)-N$ be $\gamma c$. From the equilibrium equations, we get the stiffness submatrix $\mathbf{S}_{u}$

$$
\left[\begin{array}{cccc}
-(24 \alpha+12 \beta+8 \gamma+3) & 24 \alpha & 12 \beta & 8 \gamma \\
12 \alpha & -(12 \alpha+47) & 24 & 12 \\
6 \beta & 24 & -(6 \beta+71) & 24 \\
4 \gamma & 12 & 24 & -(4 \gamma+83)
\end{array}\right]
$$

For the "upper left corner," $\alpha=7, \beta=5$ and $\gamma=3$ give, finally,

$$
\left[\begin{array}{cccc}
-255 & 168 & 60 & 24 \\
84 & -131 & 24 & 12 \\
30 & 24 & -101 & 24 \\
12 & 12 & 24 & -95
\end{array}\right]
$$

\section{A.1 Stiffness matrix for a bar with half-weight end elements}

For a bar with half-weight end elements, the procedure to determine the coefficients of the central equilibrium equations, with equilibrium equation or row number $j=m+1, \ldots, N-$ $m$, is the same as for a bar with full-weight end elements.

Going upwards from equation $m+1$, the equilibrium equation coefficients must in addition to the above adjustment (due to outgoing coefficients), also be adjusted for the half-weight end elements. Because an end element's weight is one half, the first coefficient in equation $n$ is halved accordingly. This adjustment is balanced by the diagonal coefficient which in turn is adjusted accordingly. These two adjustments follow directly from the corresponding equilibrium equation. Thus, going upwards, we adjust the end element

$$
S(n, 1)=S(n, 1) / 2
$$

The new coefficient, $S(n, 1)$, to the left in the equation, is now one half its old value, $S(n, 1)$ to the right in the equation. The diagonal coefficient is therefore balanced with the new $S(n, 1)$ value:

$$
S(n, n)=S(n, n)+S(n, 1)
$$

Note that these adjustments are row independent and can therefore be performed in any order.

Going downwards from equation $N-m+1$ to $N$, the corresponding adjustments are

$$
S(n, N)=S(n, N) / 2
$$

and

$$
S(n, n)=S(n, n)+S(n, N)
$$

Note, however, that the unique part of the stiffness matrix for a bar with half-weight end points is not symmetric. 


\section{Appendix B: Relative strain energy density values}

Relative strain energy of a bar end with $\delta=2 \Delta$ and $\alpha=3 / 2$ :

\begin{tabular}{lllllll}
\hline Point & Bond & $c$ & $\xi$ & $V$ & $c \xi V$ & $\sum c \xi V$ \\
\hline 1 & $1 \leftrightarrow 2$ & $3 / 2$ & 1 & 1 & $3 / 2$ & $5 / 2$ \\
& $1 \leftrightarrow 3$ & 1 & 2 & $1 / 2$ & 1 & \\
2 & $2 \leftrightarrow 1$ & $3 / 2$ & 1 & 1 & $3 / 2$ & $7 / 2$ \\
& $2 \leftrightarrow 3$ & 1 & 1 & 1 & 1 & \\
& $2 \leftrightarrow 4$ & 1 & 2 & $1 / 2$ & 1 & \\
i... & $i \leftrightarrow(i-2)$ & 1 & 2 & $1 / 2$ & 1 & 4 \\
& $i \leftrightarrow(i-1)$ & 1 & 1 & 1 & 1 & \\
& $i \leftrightarrow(i+1)$ & 1 & 1 & 1 & 1 & \\
& $i \leftrightarrow(i+2)$ & 1 & 2 & $1 / 2$ & 1 & \\
\hline
\end{tabular}

Relative strain energy of a bar end with $\delta=3 \Delta, \alpha=5 / 2$, and $\beta=3 / 2$

\begin{tabular}{|c|c|c|c|c|c|c|}
\hline Point & Bond & $c$ & $\xi$ & $V$ & $c \xi V$ & $\sum c \xi V$ \\
\hline \multirow[t]{3}{*}{1} & $1 \leftrightarrow 2$ & $5 / 2$ & 1 & 1 & $5 / 2$ & \multirow[t]{3}{*}{7} \\
\hline & $1 \leftrightarrow 3$ & $3 / 2$ & 2 & 1 & 3 & \\
\hline & $1 \leftrightarrow 4$ & 1 & 3 & $1 / 2$ & $3 / 2$ & \\
\hline \multirow[t]{4}{*}{2} & $2 \leftrightarrow 1$ & $5 / 2$ & 1 & 1 & $5 / 2$ & \multirow[t]{4}{*}{7} \\
\hline & $2 \leftrightarrow 3$ & 1 & 1 & 1 & 1 & \\
\hline & $2 \leftrightarrow 4$ & 1 & 2 & 1 & 2 & \\
\hline & $2 \leftrightarrow 5$ & 1 & 3 & $1 / 2$ & $3 / 2$ & \\
\hline \multirow[t]{5}{*}{3} & $3 \leftrightarrow 1$ & $3 / 2$ & 2 & 1 & 3 & \multirow[t]{5}{*}{$17 / 2$} \\
\hline & $3 \leftrightarrow 2$ & 1 & 1 & 1 & 1 & \\
\hline & $3 \leftrightarrow 4$ & 1 & 1 & 1 & 1 & \\
\hline & $3 \leftrightarrow 5$ & 1 & 2 & 1 & 2 & \\
\hline & $3 \leftrightarrow 6$ & 1 & 3 & $1 / 2$ & $3 / 2$ & \\
\hline \multirow[t]{6}{*}{ i... } & $i \leftrightarrow(i-3)$ & 1 & 3 & $1 / 2$ & $3 / 2$ & \multirow[t]{6}{*}{9} \\
\hline & $i \leftrightarrow(i-2)$ & 1 & 2 & 1 & 2 & \\
\hline & $i \leftrightarrow(i-1)$ & 1 & 1 & 1 & 1 & \\
\hline & $i \leftrightarrow(i+1)$ & 1 & 1 & 1 & 1 & \\
\hline & $i \leftrightarrow(i+2)$ & 1 & 2 & 1 & 2 & \\
\hline & $i \leftrightarrow(i+3)$ & 1 & 3 & $1 / 2$ & $3 / 2$ & \\
\hline
\end{tabular}


Relative strain energy of a bar end with $\delta=4 \Delta, \alpha=7 / 2, \beta=5 / 2$, and $\gamma=3 / 2$

\begin{tabular}{|c|c|c|c|c|c|c|}
\hline Point & Bond & $c$ & $\xi$ & $V$ & $c \xi V$ & $\sum c \xi V$ \\
\hline \multirow[t]{4}{*}{1} & $1 \leftrightarrow 2$ & $7 / 2$ & 1 & 1 & $7 / 2$ & 15 \\
\hline & $1 \leftrightarrow 3$ & $5 / 2$ & 2 & 1 & 5 & \\
\hline & $1 \leftrightarrow 4$ & $3 / 2$ & 3 & 1 & $9 / 2$ & \\
\hline & $1 \leftrightarrow 5$ & 1 & 4 & $1 / 2$ & 2 & \\
\hline \multirow[t]{5}{*}{2} & $2 \leftrightarrow 1$ & $7 / 2$ & 1 & 1 & $7 / 2$ & $23 / 2$ \\
\hline & $2 \leftrightarrow 3$ & 1 & 1 & 1 & 1 & \\
\hline & $2 \leftrightarrow 4$ & 1 & 2 & 1 & 2 & \\
\hline & $2 \leftrightarrow 5$ & 1 & 3 & 1 & 3 & \\
\hline & $2 \leftrightarrow 6$ & 1 & 4 & $1 / 2$ & 2 & \\
\hline \multirow[t]{6}{*}{3} & $3 \leftrightarrow 1$ & $5 / 2$ & 2 & 1 & 5 & 14 \\
\hline & $3 \leftrightarrow 2$ & 1 & 1 & 1 & 1 & \\
\hline & $3 \leftrightarrow 4$ & 1 & 1 & 1 & 1 & \\
\hline & $3 \leftrightarrow 5$ & 1 & 2 & 1 & 2 & \\
\hline & $3 \leftrightarrow 6$ & 1 & 3 & 1 & 3 & \\
\hline & $3 \leftrightarrow 7$ & 1 & 4 & $1 / 2$ & 2 & \\
\hline \multirow[t]{7}{*}{4} & $4 \leftrightarrow 1$ & $3 / 2$ & 3 & 1 & $9 / 2$ & $31 / 2$ \\
\hline & $4 \leftrightarrow 2$ & 1 & 2 & 1 & 2 & \\
\hline & $4 \leftrightarrow 3$ & 1 & 1 & 1 & 1 & \\
\hline & $4 \leftrightarrow 4$ & 1 & 1 & 1 & 1 & \\
\hline & $4 \leftrightarrow 6$ & 1 & 2 & 1 & 2 & \\
\hline & $4 \leftrightarrow 7$ & 1 & 3 & 1 & 3 & \\
\hline & $4 \leftrightarrow 8$ & 1 & 4 & $1 / 2$ & 2 & \\
\hline \multirow[t]{8}{*}{ i... } & $i \leftrightarrow(i-4)$ & 1 & 4 & $1 / 2$ & 2 & 16 \\
\hline & $i \leftrightarrow(i-3)$ & 1 & 3 & 1 & 3 & \\
\hline & $i \leftrightarrow(i-2)$ & 1 & 2 & 1 & 2 & \\
\hline & $i \leftrightarrow(i-1)$ & 1 & 1 & 1 & 1 & \\
\hline & $i \leftrightarrow(i+1)$ & 1 & 1 & 1 & 1 & \\
\hline & $i \leftrightarrow(i+2)$ & 1 & 2 & 1 & 2 & \\
\hline & $i \leftrightarrow(i+3)$ & 1 & 3 & 1 & 3 & \\
\hline & $i \leftrightarrow(i+4)$ & 1 & 4 & $1 / 2$ & 2 & \\
\hline
\end{tabular}

\section{References}

1. Silling SA (2000) Reformulation of elasticity theory for discontinuities and long-range forces. J Mech Phys Solids 48(1):175-209. https://doi.org/10.1016/S0022-5096(99)00029-0

2. Silling SA, Askari E (2005) A meshfree method based on the peridynamic model of solid mechanics. Comput Struct 83(17-18):1526-1535. https://doi.org/10.1016/j.compstruc.2004.11.026

3. Bobaru F, Yang M, Alves LF, Silling SA, Askari E, Xu J (2009) Convergence, adaptive refinement, and scaling in 1D peridynamics. Int J Numer Methods Eng 77(6):852-877. https://doi.org/10.1002/nme.2439 
4. Silling SA, Zimmermann M, Abeyaratne R (2003) Deformation of a peridynamic bar. Journal of Elasticity 73(1-3):173-190. https://doi.org/10.1023/B:ELAS.0000029931.03844.4f

5. Weckner O, Abeyaratne R (2005) The effect of long-range forces on the dynamics of a bar. Journal of the Mechanics and Physics of Solids 53(3):705-728. https://doi.org/10.1016/j.jmps.2004.08.006

6. Mikata Y (2012) Analytical solutions of peristatic and peridynamic problems for a 1D infinite rod. Int J Solids Struct 49(21):2887-2897. https://doi.org/10.1016/j.ijsolstr.2012.02.012

7. Madenci E, Oterkus E (2014) Peridynamic theory and its applications. Springer, New York

8. Seleson P, Littlewood DJ (2016) Convergence studies in meshfree peridynamic simulations. Computers \& Mathematics with Applications 71(11):2432-2448. https://doi.org/10.1016/j.camwa.2015.12.021

9. Chen Z, Bakenhus D, Bobaru F (2016) A constructive peridynamic kernel for elasticity. Comput Meth Appl Mech Eng 311:356-373. https://doi.org/10.1016/j.cma.2016.08.012

10. Aguiar AR, Patriota TVB, Royer-Carfagni G, Seitenfuss AB (2018) Boundary layer effects in a finite linearly elastic peridynamic bar. Latin American Journal of Solids and Structures 15(10):1-14. https://doi.org/10.1590/1679-78254337

11. Nishawala V, Ostoja-Starzewski M (2017) Peristatic solutions for finite one- and two-dimensional systems. Mathematics and Mechanics of Solids 22(8):1639-1653. https://doi.org/10.1177/10812865166 41180

12. Le QV, Bobaru F (2018) Surface corrections for peridynamic models in elasticity and fracture. Comput Mech 61(4):499-518. https://doi.org/10.1007/s00466-017-1469-1

13. Prakash N (2018) Calibrating bond-based peridynamic parameters using a novel least squares approach. J Peridyn Nonlocal Model (online ahead of print) https://doi.org/10.1007/s42102-018-0002-z

14. Ha Y, Bobaru F (2010) Studies of dynamic crack propagation and crack branching with peridynamics. Int J Fract 162(1-2):229-244. https://doi.org/10.1007/s10704-010-9442-4

15. Henke S, Shanbhag S (2014) Mesh sensitivity in peridynamic simulations. Comput Phys Commun 185(1):181-193. https://doi.org/10.1016/j.cpc.2013.09.010

16. Dipasquale D, Sarego G, Zaccariotto M, Galvanetto U (2016) Dependence of crack paths on the orientation of regular 2D peridynamic grids. Eng Fract Mech 160:248-263. https://doi.org/10.1016/j. engfracmech.2016.03.022

17. Hu W, Ha YD, Bobaru F, Silling SA (2012) The formulation and computation of the nonlocal J-integral in bond-based peridynamics. Int J Fract 176(2):195-206. https://doi.org/10.1007/s10704-012-9745-8

18. Rao SS (2017) Mechanical Vibrations in SI Units, 6th edn. Pearson

Publisher's Note Springer Nature remains neutral with regard to jurisdictional claims in published maps and institutional affiliations. 